\title{
Quantitative Untersuchung der Schweißdrüse der Zehenhaut bei den Affen
}

\author{
Von \\ Tatsuya, lizuka \\ Aus dem Anatomischen Institut der Keio Universität, Tokyo \\ (Direktor; Prof. Dr. T. Taniguchi)
}

Ich habe in der vorigen Abhandlung (Folia Anat. Japon. Bd. 25. Heft 5-6.) die Mengenverhältniße der Schweißdrüse an den 10 verschiedenen Körperteilen bei 5 Affen vergleichend-anatomisch untersucht. Es ist eine besondere Eigentümlichkeit sowohl der Handteller- als auch der Fußsohlenhaut des Menschen und Affen, daß dort, wie schon durch die Untersuchungen von Kuriki und mir hingewiesen, die reichlichere Schweißdrüse $z u$ finden ist.

Es zeigt sich das gleiche Verhältnis in der Fingerhaut wie in der Handteller- und Fußsohlenhaut, daß das Haar, die Talgdrüse und Haarbalgmuskel nicht $\mathrm{zu}$ finden sind. Ich werde hier die Befunde der Mengenbestimmung der Schweißdrüse in der Fingerhaut (vordere Extremität) bei den Affen mitteilen, und darauf sie mit diejenigen sonstiger Körperteille des Affen vergleichen.

Das mir zur Verfügung gestandene Material stammte aus den gleichen Affen, die in der letzteren Arbeit benützt wurden. Die sonstige Untersuchungsmethode war die gleiche wie in der vorigen Mitteilung. ${ }^{\text {) }}$

Im Folgenden habe ich meine Befunde tabellarisch kurz zusammengestellt (Tabelle 1-3).

Die Ausmündungszahl der Schweißdrüse in $1 \mathrm{qcm}$ Hautflächen. inhalt, ist wie in der Tabelle 1 gezeigt, je nach den Fingerarten und Affenindividuen affallend verschieden. Sie beträgt am IV. Finger 1050

1) Über die Verteilung der Schweißdrüsen bei den Affen. Okajimas Folia Anat. Jap. Bd. 25. Heft. 5-6. 1954. 
von Fall 3 und am II. Finger 3075 von Fall 3. Also beläuft sich die relative Schwankungsbreite bei den allen untersuchten Fällen und Fingern auf ca. $3-1$.

Die Reihenfolge der Größe der Ausmündungszahl ist wie folgt;

bei Fall 1: III. II. IV. I. V. Finger,

bei Fall 2: III. I. V. II. IV. Finger,

bei Fall 3: V. III. II. I. IV. Finger,

bei Fall $4:$ II. V. I. IV. III. Finger,

bei Fall 5: IV. III. I. IV. V. Finger.

Tabelle 1. Die Ausmündungszahl der Drüsen in $1 \mathrm{qcm}$ Hautfächeninhalt.

\begin{tabular}{|c|c|c|c|c|c|c|}
\hline Fälle & Fall 1 & Fall 2 & Fall 3 & Fall 4 & Fall 5 & Durchschnitt \\
\hline I. Finger & 1745 & 1918 & 2100 & 1743 & 2124 & 1926 \\
II. " & 2180 & 1393 & 2485 & 1875 & 1743 & 1935 \\
III. " & 2185 & 3075 & 2645 & 1050 & 2325 & 2256 \\
IV. " & 2015 & 1225 & 1486 & 1267 & 2725 & 1745 \\
V. " " & 1630 & 1558 & 2782 & 1846 & 1775 & 1918 \\
\hline Durchschnitt & 1951 & 1834 & 2300 & 1556 & 2138 & 1956 \\
\hline
\end{tabular}

Die Durchschnittszahl der 5 Finger eines Affens ist beim Fall 3 (2256) am großtən, dann folgen der Fall 2 (L935), Fall 1 (1926), Fall 5 (1918) und sie ist am kleinsten beim Fall 4 (1745).

Aus diesem Befunde kann man zusammenfaßend sagen, daß die Durchschnitlszahl $z$ wischen jeden Fällen keinen großen Unterschied erkennen läßt.

Das Volumen der Schweißdrüse in $1 \mathrm{qcm}$ Hautflächeninhalt ist auch je nach den Fingerarten und Affenfällen beträchtlich verschieden. Es ist am 1. Finger $(61.38 \mathrm{cmm})$ von Fall 1 am größten und äm III. Finger $(22.00 \mathrm{cmm})$ von Fall 4 am kleinsten. Also beträgt die Schwankungsbreite ca. $3: 1$.

Die Reihenfolge ist wie folgt:

Fall 1: I. IV. II. V. III. Finger,

Fall 2: III. IV. I. V. II. Finger,

Fall 3 ; V. II. IV. I. III. Finger,

Fall 4 : V. I. IV. II. III. Finger,

Fall 5: II. I. IV. III. V. F!nger. 
Quantitative Untersuchung der Schweißdrüse der Zehenhaut bei den Affen

Tabelle 2. Das Volumen der Drüsen in $1 \mathrm{qcm}$ Hautflächeninhalt.

\begin{tabular}{|c|c|c|c|c|c|c|}
\hline Fanger & I. & II. & III. & IV. & V. & Durchschnitt \\
\hline Fall 1 & 61.38 & 40.88 & 24.92 & 42.43 & 40.62 & 42.05 \\
Fall 2 & 40.88 & 39.01 & 55.18 & 53.44 & 40.14 & 45.73 \\
Fall 3 & 24.92 & 27.99 & 23.97 & 26.25 & 34.63 & 27.55 \\
Fall 4 & 41.08 & 38.45 & 22.00 & 40.09 & 49.48 & 38.22 \\
Fall 5 & 40.79 & 45.32 & 28.62 & 33.04 & 25.80 & 32.71 \\
\hline Durchschnitt & 41.81 & 36.33 & 30.93 & 37.05 & 36.13 & \\
\hline
\end{tabular}

Die Durchschnittszahl der 5 Finger ist beim Fall $2(45.73 \mathrm{cmm})$ am größten, dann kommen der Fall $1(42.05 \mathrm{cmm})$, Fall $4(38.22 \mathrm{cmm})$ und Fall $5(32.71 \mathrm{cmm})$ und sie ist beim Fall $3(27.55 \mathrm{cmm})$ am wenigsten.

Die Durchschnittszahl der 5 Affen ist am 1 . Finger $(41.81 \mathrm{cmm})$ am größten, dann folgen der IV. Finger $(37.05 \mathrm{cmm})$, II. Finger $(36.33 \mathrm{cmm})$ V. Finger $(3613 \mathrm{cmm})$ und sie ist am III. Finger am kleinsten, also ihre relative Schwankungsbreite beträgt ca $4: 3$. So läßt sich aus diesem Verhältniße verstehen, daß die durchschschnittliche Drüsenmenge in $1 \mathrm{qcm}$ Hautflächeninhalt keine deutlichen Schwankung nach den Fingerarten zeigt.

Das angenommene Volumen der einzelnen Schweißdrüse ( $\frac{\text { Volumen in } 1 \mathrm{qcm}}{\text { Ausmündungszahl in } 1 \mathrm{qcm}}$ ) schwankt $z$ wischen $0.044 \mathrm{cmm}$ (IV. Finger, Fall 2) und $0.008 \mathrm{cmm}$ (IV. Finger, Fall 5). Also ist die relative Schwankungsbreite etwas groß, sie beträgt ca $5: 1$.

Die Reihenfolge nach den Fingerarten bei jedem Fall ist wie folgt:

Fall 1: I. V. IV. II. III. Finger,

Fall 2: IV. V. I. II. III. Finger,

Fall 3: IV. V. I. II. III. Finger,

Fall $4:$ IV. V. I. III. II. Finger,

Fall 5: II. I. V. III. IV. Finger.

Das durchschnittliche Volumen des Drüsenindividuums ist beim Fall $2(0.026 \mathrm{cmm})$ am größten, dann folgen der Fall $4(0.025 \mathrm{cmm})$, Fall 1 (0.022), Fall $5(0.016 \mathrm{cmm})$ und sie ist am kleinsten beim Fall 3 $(0.013 \mathrm{cmm})$.

Das durchschnittliche Volumen von 5 Affen ist am IV. Finger $(0.025 \mathrm{cmm})$ am größten, dann kommen der I. Finger $(0.022 \mathrm{cmm})$, V. Finger $(0.021 \mathrm{cmm})$, II. Finger $(0.019 \mathrm{cmm})$ und es ist am III. Finger $(0.014 \mathrm{cmm})$ am kleinsten. 
Tabelle 3. Das Volumen der angenommenen einzelnen Drüse.

\begin{tabular}{|c|c|c|c|c|c|c|}
\hline \multicolumn{1}{|c|}{ Fälle } & Fall 1 & Fall 2 & Fall 3 & Fall 4 & Fall 5 & Durchschnitt \\
\hline Finger & & & & & \\
\hline I. & 0.036 & 0.021 & 0.012 & 0.024 & 0.019 & 0.022 \\
III. & 0.019 & 0.021 & 0.011 & 0.020 & 0.026 & 0.019 \\
IV. & 0.011 & 0.018 & 0.009 & 0.021 & 0.013 & 0.014 \\
V. & 0.021 & 0.043 & 0.018 & 0.032 & 0.008 & 0.025 \\
\hline Durchschnitt & 0.025 & 0.026 & 0.012 & 0.027 & 0.015 & 0.021 \\
\hline
\end{tabular}

Aus obigen Befunden kann man schließen, daß beim Fall 2 das Volumen der einzelnen Drüse und das in $1 \mathrm{qcm}$ Hautflächeninhalt klein sind, dagegen die Ausmündungszähl in $1 \mathrm{qcm}$ Hautflächeninhalt gross ist. Am I. und IV. Finger sind das Volumen des Drüsenindividuums und das in $1 \mathrm{qcm}$ Hautflächeninhalt größer, dagegen die Ausmündungszahl klein.

Beim III. Finger ist dieses Verhältniße ganz umgekehrt.

Aus obiger Tatsache geht hervor, daß die Größe des einzelnen Drüsenvolumens und die des Volumens in $1 \mathrm{qcm}$ Hautflächeninhalt hand in hand gehen, aber die Ausmündungszahl in $1 \mathrm{qcm}$ Hautflächeninhalt sich umgekehrt verhält.

Vergleicht man diese Befunde an den Fingerbeeren mit denen an den sonstigen Körperregionen, so ergibt sich, daß die Ausmündungszahl und das angenommene einzelne Volumen der Schweißdrüse die größte Zahl und die Menge in $1 \mathrm{qcm}$ Hautflächeninhalt die nächst größte Menge zeigen. 